\title{
RARE COEXISTENCE OF FAMILIAL MEDITERRANEAN FEVER AND FAMILIAL PARTIAL LIPODYSTROPHY: PRESENTED AS ACUTE PANCREATITIS DUE TO SEVERE HYPERTRIGLYCERIDEMIA
}

\author{
Ramazan Gen, Esen Akbay, Ümit Çınkır, Kerem Sezer \\ Department of Endocrinology and Metabolic Diseases, MERSİN, TURKEY
}

\section{INTRODUCTION}

Lipodystrophies which can be both acquired and inherited are characterized by selective loss of adipose tissue. Genetic lipodystrophies are rare, however acquired lipodystrophies can be more frequent. Partial lipodystrophy is an entity which consists of various types that can either be familial or sporadic. Progressive loss of adipose tissue from the extremities and variable loss from the body corpus and excess fat deposition in the face and neck region can be observed in partial lipodystrophies. The diagnosis is easier in women as compared to men due to the unusual muscular prominence. FMF is an inflammatory disease inherited in an autosomal recessive manner. It has two phenotypes: Type 1 is characterized by recurrent short episodes of inflammation and serositis, including fever, peritonitis, synovitis, pleuritis and rarely pericarditis. Type 2 on the other hand is characterized by amiloidoisis. FMF must be kept in mind in the differential diagnosis of acute abdomen since these patients can very often administer to emergency service with severe abdominal pain.

\section{CASE REPORT}

A 21 year old female (height $148 \mathrm{~cm}$, weight: $38 \mathrm{~kg}, \mathrm{BMI}: 17$ ) was referred to our endocrinology outpatient polyclinic from another center with the complaints of severe abdominal pain and severe hypertyriglyceridemia. Her intermittant abdominal pain complaints were present for many years which was learned to be due to familial mediterranean fever. The patient's medical history revealed diabetes since more than 10 years. For diabetes she was under subcutaneous insülin treatment. Also she was using pioglitazone for insulin resistance for nearly two years. In addition, for FMF the patient was under treatment with colchicine. Hypertriglyceridemia was present for more than 10 years just like diabetes. In this sense, not only she was being treated with phenofibrates and fish oil, but also lipid apheresis was being applied intermittently.

Physical examination of our subject revealed marked loss of subcutaneous fat from the extremities leading to prominence of muscular contours and veins, with excess fat around the face, submental and dorsocervical region giving her a Cushingoid appearance (Figure1 $a, b)$. However screening tests of $1 \mathrm{mg}$ dexamethasone suppression and cortisol level in 24 hour collected urine did not confirm Cushing's Syndrome. Cortisol level after $1 \mathrm{mg}$ dexamethasone suppression test was $38,7 \mathrm{nmol} / \mathrm{L}$ and urine free cortisol level was 122,9 $\mathrm{nmol} / \mathrm{d}$ (Normal Value 100-379 $\mathrm{nmol} / \mathrm{d}$ ). She was also suffering from hirsutism with a
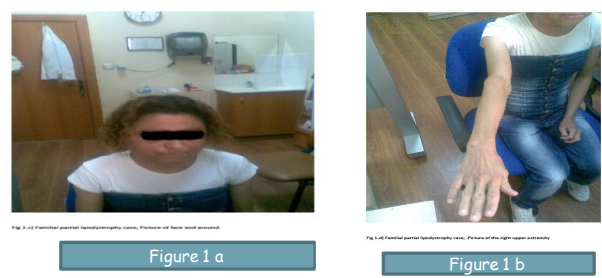
Ferriman Gallwey score of 12 and oligomenorrhea.

At the time of administration the triglyceride level (TG) of the subject was $3900 \mathrm{mg} / \mathrm{dl}$ and she had the evidence of pancreatitis attack. Abdominal CT showed edematous pancreatitis with a lipase level of $1111 \mathrm{U} / \mathrm{L}$ and an amilase level of 221U/L. Thus patient's oral intake was stopped and treatment for acute pancreatitis along with insulin infusion were startted. At the end of two days, with the appropriate treatment her complaints resolved. Nevertheless, since her TG level was $2900 \mathrm{mg} / \mathrm{dl}$, lipid apheresis decision was taken to decrease the TG levels to normal. After the first session of apheresis serum TG level was measured as $173.8 \mathrm{mg} / \mathrm{dl}$. Her anti GAD and insulin autoantibodies were negative, $c-$ peptide level was found to be $15.6 \mathrm{ng} / \mathrm{ml}$. The patient's abdominal USG revealed hepatosteatosis and hepatomegaly, whereas the CT scan of the extremities showed marked loss of subcutaneous fat. Her medical records revealed molecular diagnosis of FMF with the heterozygous mutation of E148Q which is the most seen in our country. Under the light of these results the subject was accepted as " A partial lipoditrophy case with a previous diagnosis of FMF representing with hypertyrigliseridemia and diabetes."

\section{CONCLUSION}

One rare casue of hypertriglyceridemia is the lipodystrophies. It has been shown that not all but some of the subjects with FPLD may show hypertriglyceridemia to some extent. In patients with FPLD1, subcutaneous loss of fat in the extremities along with ectopic deposition of lipids in tissues such as abdomen and liver, may lead up to insulin resistance and severe hypertriglyceridemia.

We find it interesting to present this case here because of the rare coexistence of these two diseases. 